\title{
Aproximación a la orientación de las maestrías a través de la voz de sus agentes
}

\author{
Approach to the orientation of masters through the voice of their agents \\ Silvia Elisa Castro Marcano \\ Enrique López Ramírez
}

\begin{abstract}
RESUMEN
Existe poca literatura que aborde específicamente el tema del significado de las orientaciones en el posgrado mexicano, en tal sentido, el objetivo del texto que aquí se presenta es identificar las características de las orientaciones en el posgrado y comprender el significado de dichas orientaciones desde una perspectiva enfocada en las prácticas situadas, mediante el contraste de los lineamientos normativos emanados por los organismos rectores, con la puesta en práctica de los mismos representada a través de las perspectivas de los coordinadores de distintos programas, algunos con orientación a la investigación y otros profesionalizantes, enmarcados en distintos centros universitarios de la Universidad de Guadalajara.
\end{abstract}

Palabras clave: posgrado, maestrías, procesos de formación, investigación, especialización.

\section{ABstract}

Little literature exists that specifically addresses the issue of the meaning of the Mexican postgraduate focuses, in this regard, the objective of the text presented here is to identify the characteristics of the postgraduate focuses and understand the meaning of these from a perspective based on the established practices, through the contrast of the normative guidelines emanating from the governing bodies, with the implementation the previous represented through the perspective of coordinators from different programs, some with research focus and other professionals, framed in different university centers of the Universidad de Guadalajara.

Keywords: postgraduate programs, masters, formative research process, research, specialization. 


\section{INTRODUCCIÓN}

Con el fin de caracterizar y conocer el significado de las orientaciones de los posgrados en México, la indagación que aquí se presenta tiene dos vertientes: la primera dirigida a la revisión de la literatura relacionada con el desarrollo de los posgrados en México y las orientaciones de los mismos, y la segunda, los referentes empíricos emanados del trabajo de campo, en el que se realizaron entrevistas abiertas a coordinadores de maestrías en diversas áreas disciplinares de la Universidad de Guadalajara (UDG), y que a través de sus testimonios y reflexiones exponen desde su perspectiva particular, en un contexto situado, la caracterización y significado de las orientaciones de las maestrías.

La selección de los sujetos a entrevistar tuvo como criterio el acceso y la disponibilidad de tiempo de los coordinadores en cuestión, en tal sentido, se logró realizar ocho entrevistas dirigidas a coordinadores que están a cargo de maestrías con énfasis en la investigación y con orientación profesionalizante.

La estructura del artículo tiene sus cimientos en dos pilares: el primero la revisión de la literatura referente al desarrollo histórico de los posgrados en México, por una parte, y por otra la revisión de las políticas y marcos normativos del Consejo Nacional de Ciencia y Tecnología (CONACYT) y la Subsecretaría de Educación Superior; el segundo pilar son los datos empíricos producto de las entrevistas realizadas que permiten analizar, desde el interior de los programas de maestría, cómo se llevan a la práctica las políticas y normas que se establecen en torno a la orientación de los posgrados.

\section{RUTA METOdológica}

Para el desarrollo de la investigación se adopta una perspectiva cualitativa, cuya característica principal es recuperar la subjetividad de las personas. Este enfoque se define como un constructo en el que se contempla la heterogeneidad de la realidad social mediante una serie de situaciones diversas y complejas para comprenderla e interpretarla (Cifuentes, 2011).

Silvia Elisa Castro Marcano. Profesora titular del Ministerio del Poder Popular para la Educación, Venezuela. Es Licenciada en Educación con mención en educación inicial y primera etapa de educación básica, Licenciada en Psicopedagoga con especialidad en dificultades en el aprendizaje y problemas emocionales y Doctora en Educación por la Universidad de Guadalajara. Su línea de investigación es Estilos de pensamiento y procesos de formación. Profesora de Español en los cursos propedéuticos de la coordinación de servicios estudiantiles de la Universidad de Guadalajara en el periodo 2017-2019. Correo electrónico: silviaec23@hotmail.com. ID: http://orcid.org/0000-0002-3968-6965.

Enrique López Ramírez. Investigador de Investigación, Innovación y Desarrollo Tecnológico Fornite S.A.S, Oaxaca, México. Es Profesor del Tecnológico Nacional de México/Instituto Tecnológico de Pochutla del área de Ingenierías. Es Doctor en Educación por la Universidad de Guadalajara y trabaja la línea de investigación Tecnología, resiliencia y psicología positiva. Correo electrónico: quiqueohio@hotmail.com. ID: http://orcid.org/0000-0002-7526-5199. 
El proceso inductivo propio del enfoque cualitativo es fundamental porque, a partir de elementos particulares obtenidos de la aplicación de entrevistas abiertas a coordinadores de maestrías profesionalizantes y con énfasis en la investigación de la UDG, se identificaron categorías generales que permitieron abordar el fenómeno estudiado.

Por su finalidad, la presente investigación es descriptiva-explicativa (Orozco y González, 2011), pues se pretende caracterizar las orientaciones de los posgrados en México mediante el contraste de la normatividad establecida para los programas de maestría y su operatividad, manifiesta en la ejecución de los mismos en las aulas.

Acorde con esta postura epistemológica, se utilizó como técnica la entrevista abierta puesto que permite aproximarse a una mayor amplitud de recursos, en comparación con otros tipos de entrevista de corte cualitativo. En tal sentido, de acuerdo con Del Rincón, Arnal, Latorre y Sans (1995), en este tipo de entrevista las preguntas y el orden en el que se formulan no están preestablecidos; las respuestas son abiertas, permitiendo que el entrevistado construya, a través del discurso, distintos significados conducentes a abordar el fenómeno estudiado.

Para caracterizar las orientaciones de los posgrados desde un espacio situado, consideramos que la voz de los agentes garantes de llevar la normativa establecida para dichos programas a la práctica son los coordinadores de los mismos. En tal sentido, la selección de los sujetos de estudio tuvo los siguientes condicionantes: que fuesen coordinadores de maestrías con énfasis en la investigación o profesionalizantes de la UDG en ejercicio, que tuviesen la disponibilidad de tiempo e interés para dar sus testimonios mediante entrevistas grabadas. Partiendo de estos criterios, luego de realizar visitas a diferentes centros universitarios de la UDG (CUCEA, CUCEI, CUAAD, CUCS y CUCSH), se seleccionaron ocho coordinadores que para el año 2017 estaban a cargo de maestrías profesionalizantes y con énfasis en la investigación.

Una vez finalizado el proceso de entrevistas, se analizaron los datos y se identificaron categorías generales que engloban el fenómeno estudiado, mismas que fungieron como núcleos a partir de los cuales se realizó el análisis que se expone en este artículo.

\section{El conteXto históRICO.}

\section{Breve Recorrido por la historia del posgrado en MÉXico}

Los estudios de posgrado en México son relativamente recientes tomando en cuenta que los antecedentes de la creación de la universidad en este país tienen un origen más remoto, tal y como lo reseña Marsiske (2006), quien señala que la Universidad Real y Pontificia de México, conocida en la actualidad como Universidad Nacional Autónoma de México (UNAM), fue creada en 1551 erigiéndose como la primera universidad mexicana, sin embargo, a partir de 1865 la educación superior en México empezó a impartirse en las Escuelas Nacionales debido a que la Universidad Real y 
Pontificia de México fue cerrada. No obstante, 1910 marca el año de la creación de lo que se considera como el inicio del periodo contemporáneo de la universidad en México a través de la unión de las Escuelas Nacionales en una sola institución, hecho que marca el surgimiento de la UNAM, institución que alcanzó su completa autonomía con la Ley Orgánica de 1945 (vigente en la actualidad), en la que se establecen las relaciones entre el Estado y la universidad.

La década de los cuarenta del siglo XX es señalada como el momento en el que surgió la primera aproximación de lo que hoy se conoce como el posgrado en México, específicamente en 1946, año en el que en la UNAM se creó la Escuela de Graduados, la cual estaría encargada de la coordinación de los estudios que se realizan luego de la obtención del título profesional y que fueron definidos como "altos estudios"; posteriormente, en 1956 la Escuela de Graduados fue sustituida por el Consejo de Doctorado, pero fue hasta 1967 cuando se creó el primer reglamento general de estudios de posgrados, momento en el que el Consejo de Doctorado se convirtió en el Consejo de Estudios de Posgrado.

La creación del Centro de Investigación y de Estudios Avanzados (Cinvestav) en el año 1961 fue un elemento clave para el desarrollo del posgrado en el país, puesto que dentro de sus objetivos estuvo aunar la investigación con la formación de cuarto nivel, en distintas disciplinas y áreas. Posteriormente, en 1970 se creó el Consejo Nacional de Ciencia y Tecnología (CONACYT), el cual a través del otorgamiento de becas fue un motor que impulsó la creación, desarrollo y fortalecimiento de los programas de posgrado (Arredondo, Pérez y Morán, 2006).

Así mismo, a finales de los años setenta, la Secretaría de Educación Pública (SEP) creó la Subsecretaría de Educación Superior e Investigación Científica (SESIC), cuya finalidad también fue el apoyo y la promoción del posgrado. Para 1978 la Asociación Nacional de Universidades e Institutos de Enseñanza Superior (ANUIES), creada en el año 1950, hizo un esfuerzo conjunto de la mano del SESIC y desarrollaron el Plan Nacional de Educación Superior con el fin de impulsar y promover nuevos programas de posgrado, ya que la oferta de este nivel de estudio era muy escasa a nivel nacional, por lo que los profesionales que quisieran darle continuidad a su formación académica debían acudir a universidades en el extranjero.

Esta dupla marcó el inicio de la coordinación y vinculación con entes nacionales, estatales, regionales e institucionales, que se constituyó como una manera de vincular a las universidades públicas con la SEP y la ANUIES, y así contrarrestar el rápido y desordenado crecimiento de la matrícula a nivel de posgrado. Este plan conjunto (SEP-ANUIES) duró diez años, de 1978 a 1988, y en el gobierno de Carlos Salinas de Gortari la SEP de alguna manera excluyó a la ANUIES de la toma de decisiones referentes a las instituciones universitarias públicas (Arredondo y Navarro, 2012).

En el año 1991 el Conacyt creó el Padrón de Programas de Posgrado de Excelencia, el cual fue implementado para otorgar recursos a los programas de posgrados 
siempre y cuando estos estuviesen registrados en dicho padrón, y para ello se establecieron diversos requerimientos a los que se debían apegar las instituciones y que, a su vez, funcionaron como mecanismos para establecer controles de calidad en la oferta académica de los posgrados. Para el año 2000 el Padrón de Programas de Excelencia llegó a acreditar 405 programas de posgrado y en el 2001, tras ciertos ajustes, se transformó en el Programa de Fortalecimiento al Posgrado Nacional, que de acuerdo a los planes estratégicos de desarrollo institucional se enfocó en el mejoramiento de los programas y marcó la incorporación de las maestrías profesionalizantes al Programa de Fortalecimiento del Posgrado Nacional (Serna y Pérez, 2012).

\section{Los estudios de Posgrado en México}

La organización actual de los estudios de posgrado en México es el resultado de su desarrollo histórico y del conjunto de políticas materializadas en la creación y consolidación de distintos organismos que se enfocaron a desarrollar y fortalecer al posgrado, evidencia de ello es la descripción de su configuración histórica, descrita a grandes rasgos en el apartado anterior. Al respecto, Arredondo y Navarro (2012) plantean que "los estudios de posgrado en México representan un universo de gran complejidad y heterogeneidad, en parte debido a la historia del país, y en particular a la historia de la educación superior" (p. 13).

El posgrado en México en el siglo XXI se caracteriza por la poca cobertura en contraste con el crecimiento de la matrícula, la concentración de los posgrados en la capital y en las principales ciudades del país, la variabilidad de la calidad entre instituciones y, dentro de estas, incremento en la realización de estancias en el extranjero, aumento de la oferta de programas en instituciones privadas, la utilización de la demanda como elemento que genera una marcada mercantilización del posgrado, baja eficiencia terminal, asignación de salarios que no concuerdan con el nivel de preparación de los egresados, inversión estatal en investigación baja a nivel público y muy escasa en el nivel privado (Luchilo, 2010).

El Programa Nacional de Posgrados de Calidad (PNPC), como política pública, es la evidencia más notoria del interés en incrementar la calidad del posgrado. El mismo se constituyó como un esfuerzo conjunto desde 1991 por parte del ConACYT y la Subsecretaría de Educación Superior de la Secretaría de Educación Pública.

\section{Las orientaciones en el posgrado}

Una de las formas en las que se clasifican los programas de posgrado en México son las orientaciones de los mismos. El PNPC reconoce la orientación a la investigación y la profesional en el nivel de posgrado.

Partiendo de la lectura del Marco de Referencia para Evaluación y Seguimiento de Programas de Posgrados Presenciales desarrollado por ConACYT-SEP (2015), aun 
cuando no se explicite, se puede interpretar que la orientación de los programas de posgrado refleja los fines y el camino a seguir para la formación de recursos humanos; en tal sentido, en las orientaciones estarían implicados el currículum de cada programa y los procesos de formación que se producen en el interior de cada uno de ellos.

Las orientaciones en los posgrados son reconocidas tanto desde el marco de referencia antes mencionado como en el Reglamento general de posgrado de la Universidad de Guadalajara (2004). En el Marco de Referencia para Evaluación y Seguimiento de Programas de Posgrados Presenciales (ConACYT-SEP, 2015) no se ofrece una definición propiamente dicha en el documento que se enfoca en las orientaciones de los posgrados; al respecto en el documento se expone textualmente que

Los Programas de Posgrado con Orientación a la Investigación se ofrecen en los niveles de especialidad, maestría y doctorado en las diferentes áreas del conocimiento. Los Programas de Posgrado con Orientación Profesional se ofrecen en los niveles de doctorado, maestría y especialidad, con la finalidad de estimular la vinculación con los sectores de la sociedad [p. 9].

De inicio, se observa que en este marco de referencia se especifican los niveles a los que se aplican las orientaciones y esta exposición inicial del documento únicamente se refiere a la finalidad de los programas de posgrado con orientación profesional, pero no menciona la finalidad de aquellos posgrados con orientación a la investigación sino hasta más adelante, cuando se exponen los rasgos y referentes de las orientaciones.

Tomando como referencia el Reglamento general de posgrado de la Universidad de Guadalajara (2004), se puede entender que las maestrías que brindan una formación enfocada en los procesos de investigación tienen como objetivo, de acuerdo al reglamento antes mencionado, "proporcionar conocimientos en una disciplina o área interdisciplinaria, profundizando en los aspectos teóricos, metodológicos o tecnológicos básicos para la investigación y generación de conocimientos” (p. 1), y las maestrías profesionalizantes tienen como objetivo "proporcionar conocimientos en una disciplina o área interdisciplinaria, profundizando en los aspectos teóricos, metodológicos o tecnológicos para ponerlos en práctica en el desempeño profesional" (p. 1).

Para los fines de este artículo se requiere conocer un poco más las características de las orientaciones de los posgrados, más específicamente de las maestrías, y en este apartado se abordará este punto desde la normatividad emanada por el CONACYT, en la que de manera muy general se caracterizan estos programas. En tal sentido, se toma como base para esta caracterización el apartado referente a los principales rasgos de los posgrados profesionales, desarrollado en el documento correspondiente al Marco de Referencia para Evaluación y Seguimiento de Programas de Posgrados Presenciales (CONACYT-SEP, 2015) emanado por el CONACYT y la Subsecretaría de Educación Superior. 
Un punto que vale la pena destacar es que el documento antes mencionado hace referencia específica a los doctorados y sólo en unos cuantos puntos se refiere a las maestrías; partiendo de esta observación ya se nota poca claridad en cuanto a la definición de las orientaciones para el nivel de maestría, lo que de alguna manera podría estar causando confusión en los usuarios de estos lineamientos normativos.

En cuanto a la admisión de estudiantes, el marco de referencia establece que cada programa de maestría debe contar con un proceso de admisión riguroso que garantice los conocimientos previos de los aspirantes, este rasgo es exactamente igual para las dos orientaciones, este criterio flexibiliza las posibilidades de admisión en cada programa y deja a criterio e interpretación de quienes los diseñan establecer cuáles son los conocimientos que se requieren para formar parte de un programa de posgrado en particular.

En cuanto al perfil de egreso, se establecen diferencias en las orientaciones. En el caso de las maestrías profesionalizantes se plantea como propósito "profundizar en las habilidades y competencias de un campo profesional para atender la demanda potencial del mercado laboral y de impacto inmediato al sector profesional" [p. 9]. En lo que se refiere a la orientación a la investigación "el programa tiene como propósito la formación de investigadores críticos y creativos a través de investigaciones originales" [p. 13]. En tal sentido, se observa que las competencias que desarrollan los estudiantes en una maestría con orientación profesionalizante guardan relación con las demandas del mercado laboral, y el objetivo de los programas con énfasis en la investigación está dirigido al desarrollo de habilidades para la investigación, que conllevan a la formación de investigadores.

Una diferencia fundamental entre las dos orientaciones está centrada en el tipo de trabajo terminal que se ha de realizar en cada una, en este particular el marco de referencia sí establece lineamientos específicos tanto para la especialización como para la maestría y el doctorado; al respecto se menciona que

El trabajo terminal considera memorias, proyecto terminal, informe de actividad profesional o tesinas y éstos deben de estar sistemáticamente asociados a trabajos realizados en estancias en laboratorios de investigación, centros de investigación y desarrollo en las empresas, o diferentes lugares relacionados con el ámbito socioeconómico del posgrado como hospitales, centros de educación, estructuras de gobierno, tribunales de justicia, etcétera [p. 11].

En el caso de las maestrías con énfasis en la investigación, se establece como única forma de trabajo terminal la tesis. Al inicio del párrafo anterior se calificó como fundamental para esta indagación la diferencia entre el tipo de trabajo que se espera en cada orientación, ya que dicho trabajo constituye la mayor tarea o desafío al que tiene que hacer frente el estudiante que realiza una maestría.

García (2015) expone en su tesis doctoral que la orientación profesionalizante tiene un significado multiforme, sui géneris, que se configura en el interior de cada 
programa; al respecto la autora afirma que "la orientación de los posgrados sólo es una ventana al contenido del currículum, que tendrá que reflejar con mayor claridad de qué manera se interpreta la orientación profesionalizante" [p. 21]; en este particular caso, García coincide con la percepción de quienes escriben este artículo, pero, desde la perspectiva de este texto, también parecería atribuible a la orientación a la investigación un significado multiforme que va directamente relacionado con el currículum, funcionamiento y con las concepciones que se tienen en el interior de cada programa de maestría acerca de lo que significa hacer investigación científica. Esta afirmación tiene su sustento en las evidencias empíricas que se exponen en el siguiente apartado.

\section{Contextualización de las maestrías con orientación profesionalizantes y con orientación a la investigación. De la normatividad a las prácticas cotidianas, un acercamiento a través de la voz de algunos de sus agentes}

Debido a la poca literatura y escasa claridad en torno a las diferencias entre los programas de posgrado con énfasis en la investigación y los de orientación profesionalizante, y con la finalidad de obtener una caracterización de las orientaciones de los posgrados, además de un acercamiento hacia la comprensión del significado de la orientación de los programas desde su contexto, el presente apartado se enfoca en caracterizar y contextualizar las orientaciones de los posgrados desde la perspectiva de sus coordinadores, las cuales fueron capturadas a través de la realización de entrevistas abiertas a estos agentes.

Aun cuando, tal y como se señala en el apartado anterior, existe un marco normativo emanado del PNPC en el que se contemplan las orientaciones de los posgrados, desde la perspectiva de este texto, se requiere comprender qué se entiende por "profesionalizante" y por "énfasis en la investigación", desde el contexto en que se llevan a cabo las prácticas de formación.

Algunos de los testimonios de los coordinadores son concordantes con lo que se establece desde la normatividad de las orientaciones de los posgrados y de los lineamientos emanados desde el PNPC, pero otros funcionan como contraejemplos (actuaciones fuera de la norma) a lo que se espera del funcionamiento de un programa profesionalizante o con énfasis en la investigación, desde el marco de referencia que reglamenta los posgrados de calidad en México.

Los aspectos abordados a través de las opiniones y reflexiones de los coordinadores fueron muy variados, para efectos de este artículo se presentarán aquellos elementos del discurso que ofrecen pistas sobre la caracterización de las orientaciones de los posgrados, con el fin de poder identificar las diferencias en las orientaciones, más allá de los documentos normativos.

En cuanto a las diferencias entre la orientación profesionalizante y la orientación a la investigación se evidencia que algunos coordinadores en un primer momento las 
establecen desde la norma, en tal sentido, hay opiniones que se apegan a las normativas del PNPC, tal y como lo expone el coordinador de uno de los programas situados en el CUCEI cuando expresa que

La diferencia fundamental está en que la orientación a la investigación está dirigida a la generación de conocimientos y la profesionalizante a la aplicación de los mismos [EC1C1]. ${ }^{1}$

Esta opinión fue generalizada, es decir, la mayoría de los sujetos entrevistados expuso que la aplicación y generación de conocimientos sería la diferencia fundamental entre ambas orientaciones, sin embargo también reconocieron que la investigación está implicada en ambas orientaciones y lo que marca las fronteras entre ambas es el tipo de investigación que emana de cada programa.

Ambos programas tienen un trasfondo de investigación. Los programas profesionalizantes te llevan más a una especialización en la parte profesional, en el hacer. Los que tienen énfasis en la investigación hacen investigación de fronteras, hacen investigación pura. Hago una diferenciación en el tipo de investigación que se hace, los profesionalizantes se enfocan en el desarrollo profesional del estudiante en su intervención en la práctica en los sectores públicos y privados [EC2C2].

Otro elemento diferenciador es el que se refiere a la motivación de los estudiantes que se acercan a un programa de posgrado. En relación con esto, uno de los coordinadores de una maestría profesionalizante expresó que

El receptor del servicio es totalmente diferente de un programa a otro, entendiendo como que ofrecemos un servicio [...] la persona que se acerca a un programa profesionalizante tiene una motivación totalmente diferente a los que se acercan a una maestría con énfasis en la investigación [...] por lo general las profesionalizantes están hechas para personas que están ya en el ámbito laboral y que las competencias que buscan adquirir son para fortalecer su práctica profesional [EC3C].

Este comentario alude a la formación de posgrado como un servicio que se ofrece, en el que el bien que se oferta es la capacitación de aquel que decide adquirirlo, pero esta formación que busca el estudiante da la impresión de estar más relacionada con los beneficios laborales que con los del aprendizaje; cabe preguntarse, si se asumen las motivaciones de los estudiantes desde esta perspectiva, ¿dónde quedan las motivaciones relacionadas con el crecimiento personal y académico?

Por otro lado, las motivaciones de los estudiantes hacia las maestrías con orientación a la investigación, también desde la perspectiva de quien coordina un posgrado profesionalizante, están sujetas a los espacios donde se desempeñan laboralmente los aspirantes, sin embargo en este caso el comentario da a entender que este tipo de maestría no generaría interés en quien no se encuentre relacionado con el ámbito de la investigación y que en este tipo de programa el aprendizaje y la formación que se

1 El código que se asigna al final de cada viñeta corresponde al coordinador entrevistado y al centro universitario al que pertenece. 
suscitan en su interior no tienen utilidad alguna en el campo laboral. En tal sentido, el significado que se está dando desde esta opinión a la formación para la investigación y a la profesionalizante sólo se enfoca en los beneficios laborales que luego de la obtención del grado de maestro van a alcanzar sus estudiantes.

[...] un programa en investigación por lo general lo buscan personas que trabajan ya dentro de una institución donde se hace investigación. ¡Si yo tomo una maestría en investigación y no estoy ligado a la investigación no va a servir de nada! [...] El tipo de alumnos que tenemos están muy enfocados en adquirir competencias para mejorar su condición laboral, lo hacen para obtener un mejor puesto, lo que no pasa en investigación a menos que estés ya dentro de una institución universitaria [EC3C3].

Desde la perspectiva del coordinador de una maestría con énfasis en la investigación, las diferencias entre las orientaciones son abordadas de manera muy distinta, lo que queda reflejado en los siguientes comentarios:

La diferencia es que una maestría que está orientada a la investigación va a formar gente con juicio propio y crítico, sin embargo, por lo que he leído, esto no está muy explícito en los programas profesionalizantes [EC4C4].

Le llaman tesis, pero no es tesis, porque no tiene la metodología de una tesis de investigación y entonces se observa una desvinculación en este tipo de trabajos y observamos en proyectos de intervención planteamientos de hipótesis y esto no es congruente [EC5C5].

Estos planteamientos enuncian los beneficios intrínsecos relativos al desarrollo académico, muy vinculado con las habilidades para la investigación que propone Moreno (2002) en el caso del primer comentario, en el que la diferencia está marcada por el desarrollo del pensamiento crítico en los programas con énfasis en la investigación. Así mismo se alude a la tesis y el rigor con el que esta se construye como una diferencia entre las orientaciones, ya que se señala que en los programas profesionalizante se entiende por tesis algo distinto a lo que esta representa en un programa de orientación profesionalizante.

Otra perspectiva, ofrecida por un coordinador a cargo de un programa con orientación a la investigación, señala que solo existe una orientación posible para un programa de maestría, y esta es la orientación a la investigación, ya que, desde la visión de este coordinador, los programas profesionalizantes deberían ser especializaciones:

Yo nunca estuve de acuerdo con las maestrías profesionalizantes; para mí eso es una especialidad donde se enseñan conocimiento aplicado a resolver problemas prácticos, pero no se enseña pensamiento científico [EC5C5].

En este caso lo que estaría estableciendo una diferencia sería el desarrollo del pensamiento crítico; desde esta postura se podría inferir, en primera instancia, que en los proyectos que se desarrollan desde los programas con orientación profesionalizante no se pone de manifiesto el pensamiento metodológico y todos los procesos 
cognitivos que desde éste se movilizan, sino que más bien se enseña a aplicar conocimientos a situaciones prácticas.

Otro tópico que salió a relucir en las entrevistas, es el que se refiere a las fronteras difusas entre la orientación profesionalizante y la orientación a la investigación, al respecto se encuentran comentarios como los siguientes:

No hay en el reglamento un concepto o especificaciones prácticas de cómo han de ser los programas profesionalizantes y con énfasis en la investigación. Hace falta un reglamento más específico que dé las pautas para ambas orientaciones [EC2C2].

He revisado los marcos de referencia, me sorprende porque no está claro del todo... Para mí, los profesionalizantes son para aplicar lo que saben, para ejecutar, sin revisar mucho estas perspectivas; sigo pensando que los de investigación serían el fundamento para el desarrollo y avance en la actual sociedad del conocimiento [EC4C4].

Los comentarios señalan la falta de claridad del reglamento en lo que se refiere a las orientaciones de los programas. Al revisar el Marco de Referencia para Evaluación y Seguimiento de Programas de Posgrados Presenciales (ConacyT-SEP, 2015) se nota un vacío en cuanto a cómo implementar la orientación profesionalizante o a la investigación en las maestrías.

De acuerdo con García (2015), los marcos de referencia del CONACY'T cada día han ampliado más la información relacionada con las orientaciones, sin embargo, explícitamente en los apartados del marco de referencia denominados "Principales rasgos de los programas de posgrados de orientación profesional" (p. 9) y "de orientación a la investigación" (p. 13), el documento alude en su preámbulo a los programas de doctorado tanto profesionalizantes como con orientación a la investigación y no hace una referencia directa a las maestrías, y en la tabla donde se especifican los rasgos y referentes de estos programas en cada orientación se habla en líneas generales de los posgrados, pero las notas de pie de página hacen referencia directa a los doctorados y sólo dos criterios se dirigen específicamente a las maestrías, el primero denominado "Estructura del trabajo terminal", correspondiente a la orientación profesional, en el que se señala el tipo de trabajo final que deben elaborar los estudiantes para alcanzar el grado de maestros, y el segundo criterio, "Tesis de maestría y especialidad", correspondiente a la orientación a la investigación, en el que se establece la tesis como el tipo de trabajo para la obtención del título correspondiente.

Lo antes expuesto indica que una de las diferencias fundamentales entre ambas orientaciones es el tipo de trabajo que deben elaborar los estudiantes como producto final de la maestría, pero la falta de especificidad en estos criterios deja abierta la posibilidad a muchas interpretaciones y esto se ve evidenciado en opiniones como las expuestas por los entrevistados, que son un reflejo de la falta de diferenciación específica entre las orientaciones. 
En el mismo orden de ideas, relacionadas con las fronteras difusas entre las orientaciones, se encontraron opiniones como las siguientes, emanadas en el primer caso por el coordinador de una maestría con orientación a la investigación y en el segundo por el coordinador de una maestría profesionalizante, ambos testimonios ejemplifican la interpretación de los coordinadores sobre las orientaciones de sus programas:

Esta maestría de alguna forma es profesionalizante, considero que esta tiene muchos elementos de esto [EC1C1].

Hicimos la solicitud ante el CONACYT y no entramos. Nos rechazaron porque el programa tiene todos los rasgos de investigación, pero nosotros planteamos que era un programa profesionalizante [EC3C3].

Otro tópico que salió a relucir con frecuencia en las entrevistas realizadas fue el referente a la simulación como estrategia para la permanencia en el PNPC, la cual se presenta de diferentes maneras, que se pueden ejemplificar a través de los testimonios de los coordinadores entrevistados; a continuación se exponen algunos de ellos:

Les decimos a los muchachos que CONACYT nos está pidiendo que sea tesis pero en realidad es un proyecto de intervención. Nosotros internamente lo vamos a manejar como proyecto de intervención, pero se va a presentar en la portada como tesis porque es lo que quiere ver el CONACYT, pero para nosotros un proyecto de intervención es una investigación, esto es porque los temas tienen que ver más con la práctica profesional, en tal sentido la tesis de los muchachos son proyectos de intervención [EC3C3].

Ocupamos que los números correspondan a las exigencias del CONACYT y ahí entra la perversión que genera este organismo al empujarnos a aceptar alumnos por cumplir con las normativas y para que los profesores puedan obtener los estímulos necesarios y esto crea en los estudiantes la idea de que pueden egresar por la simple necesidad que tiene el programa de la eficiencia terminal [EC1C1].

Es un juego medio perverso ya que nosotros tenemos que fingir cosas que realmente no pasan como el hecho de ocultar que los alumnos trabajan, por ejemplo, no debería ser así, pero así lo hacemos [EC3C3].

A partir de las dos primeras opiniones se puede deducir que los requerimientos exigidos por el CONACYT pueden producir el efecto contrario para el que fueron creados, es decir, se establecen lineamientos que desde el papel parecen fomentar la calidad de los programas, así como los objetivos para ampliar la cobertura del posgrado, pero en la práctica parecen incitar a la simulación. Llama la atención de estos testimonios el uso de la expresión "juegos perversos" y "perversión” ya que de alguna manera señalan intencionalidad por parte del CONACYT y aceptación por parte de los programas. Estos mecanismos de simulación señalan violaciones a la ética, producto de las presiones ejercidas por el sistema; con respecto a este tipo de conductas, Hirsch (2012) expone, refiriéndose a la simulación, que “...este tipo de acciones constituye 
un grave problema que debe resolverse por las consecuencias que acarrea para las universidades y para la comunidad científica” [p. 142].

Pero la simulación no opera únicamente desde las acciones de la administración de la coordinación de algunos de los programas abordados, también se evidenció la simulación en algunas acciones de los docentes, señaladas por los coordinadores entrevistados. A continuación se citan algunos ejemplos de este tipo de conducta no ética:

Los profesores muestran una tendencia a aceptar la dirección de tesis de muchos alumnos para obtener las credenciales necesarias para el SNI, aun cuando en algunas ocasiones no muestran el compromiso para guiar la investigación de los alumnos que asumen [EC1C1].

Aunque dentro de este departamento hay muchos profesores investigadores la producción científica es muy baja, se trabaja mucho y dicen que se trabaja mucho, pero al final de cuentas los números son fríos; si revisas te das cuenta que hay muy pocas publicaciones de calidad, en eso veo una debilidad [EC3C3].

En el primero de los casos se evidencia que los intereses personales de los docentes investigadores sobrepasan las conductas éticas que se esperan de la actuación de quien asume la tarea de ser facilitador de procesos de aprendizaje. En el segundo caso se pone de manifiesto la simulación relacionada con la producción científica en cuanto a la falta de profundidad y de rigor de las investigaciones que se publican; al respecto Barba, González y Barba (2014) afirman que "la necesidad de publicar puede transformar por completo la forma de investigar, al desarrollar proyectos que puedan producir resultados de forma rápida" (p. 130). Es importante destacar que el análisis de estos testimonios no pretende ser una generalización de las actuaciones de los programas abordados ni de sus agentes.

Otro tópico que salió a relucir a partir de las opiniones de los coordinadores entrevistados fue el que se refiere a las orientaciones de los posgrados y su impacto en la incorporación y/o permanencia en el campo laboral; con respecto a este se emitieron opiniones que hasta cierto punto se contraponen, tal es el caso de dos entrevistados que hablan, en el primer caso, de la inserción laboral que es favorecida por la realización de una maestría con orientación a la investigación, y en el segundo se expone que la realización de una maestría con énfasis en la investigación sería un impedimento para la permanencia e ingreso en el campo laboral.

Los alumnos que hacen maestría en Economía (investigación) se ubican muy fácilmente en el campo laboral y aun estando dentro del programa reciben propuestas laborales por parte de organismos muy importantes como el Banco Mundial [EC2C2].

[...] hay pocos egresados en la licenciatura en Cultura Física y Deporte que trabajan en investigación, entonces yo creo que debemos ofrecerles una maestría que les mejore sus condiciones laborales en el campo de trabajo, si la ofrecemos en investigación [refiriéndose a la maestría] el profesional de cultura física va a venir y decir, por ejemplo, “yo trabajo en el Gimnasio VIvo 47, voy a venir contigo a hacer una maestría en investigación y me voy a trabajar en el gimnasio a seguir 
atendiendo gordos en la banda sin fin", entonces ni al estudiante ni al gimnasio les va a servir, ni les va a interesar que el profesional haga una maestría con énfasis en la investigación [EC3C3].

Las opiniones antes expuestas señalan que la orientación de una maestría puede favorecer o no a sus estudiantes dependiendo de la disciplina en la que se encuentren ubicados; de tal manera, hay disciplinas en las que se valora más la adquisición de habilidades para la investigación y en otras las habilidades prácticas referentes a una profesión.

En otros casos, como el que se ilustra a continuación, el estudiante desde antes de ingresar al programa de maestría debe estar trabajando en su área profesional porque de lo contrario no podrá tener acceso al trabajo de campo que se requiere en las investigaciones que se desarrollan dentro de la maestría; esta situación se alude en el siguiente ejemplo, en el cual la burocracia y los filtros institucionales se hacen presentes en la práctica, aun cuando no estén expresos en ninguna normativa:

El alumno debe estar inmerso en un medio hospitalario porque es muy difícil hacer investigación clínica si no estás en la plantilla de un medio hospitalario porque nada más por la pura procedencia institucional no te van a aceptar, por ejemplo, si intentas ingresar al IMSS para hacer una investigación y estás vinculada con otra institución, incluyendo la universidad, es indicativo de que no vas a entrar; no sé si eso estará escrito pero la experiencia nos ha dicho que es así [EC6C3].

Otro elemento destacable en las entrevistas realizadas es el que se refiere a las particularidades de los programas de acuerdo a su funcionamiento interno, este tópico en particular alude al contexto propiamente dicho de los programas en cuanto a su funcionamiento. $\mathrm{Al}$ respecto los coordinadores expresaron que:

En la práctica los programas pueden tener dos modalidades, a los de tiempo completo los quieren enfocar más a la investigación aun cuando su orientación sea profesional por lo que se deben enfocar más a la vinculación con el sector público y privado, pero en esto los coordinadores deben tener más claridad en los objetivos de la orientación [EC2C2].

Una de las grandes carencias en este programa es el manejo del inglés que en otros programas no es tan notorio, es como que los muchachos de esta carrera creen que vienen a divertirse, a jugar y hacer deportes y además a obtener un título universitario y no agarran con tanta seriedad y formalidad sus estudios en el aspecto teórico, a diferencia de un alumno de medicina o de psicología, tiene un nivel altísimo de inglés y de compromiso con el programa [EC2C2].

Para el egreso se piden al menos dos ponencias o un artículo, los estudiantes prefieren las ponencias, creo que esto obedece a que es menos rigurosa la participación en los congresos [EC5C5].

La diversidad en las exigencias y en el funcionamiento de los diferentes programas salió a relucir en muchas de las opiniones de los entrevistados, no sólo en las que se exponen en los fragmentos seleccionados, las mismas dan cuenta de las diferencias que existen en la formación que pueden originarse por las disciplinas, por el nivel de compromiso y las habilidades de los estudiantes, por las características del centro universitario en el que se encuentran. 
Las estrategias de formación en la orientación profesionalizante y en la orientación a la investigación fue otro de los tópicos que se hizo presente en las entrevistas realizadas; al respecto los coordinadores señalaron elementos que dan pistas de las tareas, actividades y contenidos que se abordan en el interior de los programas de las maestrías que coordinan. Con el fin de sistematizar las opiniones emitidas, las mismas se organizaron de acuerdo a ciertos ejes generadores como la enseñanza de contenidos, las vinculaciones, las tutorías y los coloquios.

Nosotros tenemos un primer curso que se llama Metodología de Investigación y empezamos por sentar las bases para que logren diferenciar lo que es un conocimiento empírico y un conocimiento científico, se les enseñan los diferentes tipos de investigación para darles una visión general [...] los alumnos no le encuentran muchas veces el sentido a aprender las estructuras conceptuales de la ciencia y por ello hacemos mucho énfasis sobre todo en la vinculación de los elementos teóricos con los prácticos [EC5C5].

En cuanto a las tutorías se evidenciaron en las entrevistas maneras muy diversas de implementarlas en la práctica; al comparar los programas entre sí, en algunas maestrías sólo se contempla la figura del director de tesis, en otras las del tutor, director y codirector, y en otras estas etiquetas son sinónimos entre sí.

Cuando entra el alumno le asignamos un tutor, director y asesor para estancia de vinculación, él debe ver el proyecto en el lugar donde se realiza la práctica [EC3C3].

Tenemos un sistema tutorial a nivel licenciatura, pero esto no está consolidado a nivel del posgrado y esto debería ser una continuidad. El director debe estar implicado en la práctica y en la investigación que hace el estudiante [...] Algunos programas en este centro universitario asignan a los estudiantes tutor y director de tesis, sobre todo en los programas a distancia, el tutor cumple el rol de orientador, psicólogo, consejero, y es fundamental para evitar la deserción. En los programas presenciales el tutor está presente desde el primer semestre, y luego se asigna el director de tesis que acompaña al estudiante a partir del segundo semestre [EC2C2].

En cuanto a las actividades y tareas que se desarrollan dentro de los programas, desde la perspectiva de sus coordinadores, que en el caso de los entrevistados todos cumplen funciones de docencia, se evidenció un abanico de estrategias que, desde el punto de vista de éstos, son las que fortalecen la consolidación de las habilidades que se esperan de un estudiante que realiza un programa profesionalizante o con orientación a la investigación; entre estas actividades destacaron las prácticas profesionales, las vinculaciones con expertos en la investigación y con las instituciones donde se realizan las prácticas, la incorporación de los alumnos a grupos de investigación y los coloquios.

Dependiendo de la naturaleza del programa se establecen las vinculaciones y el tipo de dependencia en la que se realiza. El proyecto de investigación tiene que estar vinculado con la práctica. Las prácticas tienen que tener un propósito, hoy es así, antes no funcionaba del todo de esta manera, pero estos son los ajustes que han incrementado la calidad de los programas [...] Los estudiantes 
que están en programas con orientación a la investigación están más en contacto con investigadores y ese contacto es una de las maneras en las que formamos para la investigación [EC2C2].

Tal y como se refleja en la cita anterior, las actividades que se desarrollan en los espacios con los que se vinculan los estudiantes parecieran ser las que configuran las orientaciones de los programas en el hacer; en tal sentido, el énfasis está en el contacto con el campo en el que se inserta el estudiante en su proceso de formación. En los programas profesionalizantes este campo está situado en las empresas y espacios laborales, y en el caso de los programas con énfasis en la investigación en la inserción de los estudiantes a grupos de investigación y en la construcción de un objeto de estudio.

Con respecto a la socialización del conocimiento, el coloquio, conocido también como foro en otros programas, es la actividad más recurrente dentro de los programas de maestría en ambas orientaciones, sin embargo esta práctica tiene lineamientos distintos en cada programa en cuanto a la formalidad, momento del programa en el que se hace, tiempo de duración; entonces, de acuerdo a los entrevistados, en algunos casos el coloquio es abierto al público en general y en otros es únicamente para el estudiante, su director y sus lectores; en uno de los programas esta actividad se realiza a partir del segundo semestre, en la mayoría se hace desde el primer semestre, en otros se realiza dos veces por semestre. Casi todos los coordinadores entrevistados calificaron este tipo de práctica como beneficiosa, a excepción de un coordinador que expuso que le parecía poco útil para los objetivos del programa que coordina, así como para los intereses de sus estudiantes.

Es una experiencia buena y enriquecedora, en algunos casos los coloquios se hacían dentro de la clase porque la presencia de los demás compañeros es muy enriquecedora. En el pasado para algunos programas no era una práctica recurrente, actualmente sí [EC2C2].

No dio resultado hacer coloquios dentro del programa ya que ellos [refiriéndose a los estudiantes] están acostumbrados a asistir a congresos nacionales e internacionales por lo que los coloquios les parecían algo muy básico [EC1C1].

Los coordinadores de maestrías con énfasis en la investigación destacaron la importancia de la escritura de artículos y la participación en congresos como prácticas beneficiosas en la formación de investigadores:

$\mathrm{Al}$ egresar esperamos que tengan por lo menos dos publicaciones realizadas durante el trayecto del programa, es un requisito interno. Que sean capaces de hacer difusión del conocimiento, que asistan a un congreso y que hagan una presentación de tesis en la que empleen un lenguaje técnico profundo y que los trabajos de tesis estén sustentados con una base matemática que los respalde [EC6C3].

La maestría para mí es preparación para que los estudiantes puedan escribir un artículo, aunque esto suene crudo [EC1C1]. 
Otro elemento que apareció como una constante en las entrevistas realizadas fue el que se refiere al perfil de ingreso de los estudiantes, que en cierta medida pareciera configurar las prácticas que se realizan posteriormente a lo largo del programa como actividades remediales tendentes a solventar las carencias con las que ingresan los estudiantes a la maestría. La mayoría de las deficiencias de los estudiantes, señaladas por los coordinadores, hacen referencia a vacíos en la formación que recibieron en el pregrado y a la poca claridad que tienen en relación con las orientaciones de las maestrías que seleccionan para dar continuidad a su formación académica; en otros casos se señalan las competencias con las que vienen los estudiantes como un elemento que se toma en cuenta para el ingreso a los programas. A continuación se exponen algunas de las expresiones de los entrevistados que ilustran lo antes expuesto:

La desorientación con la que llegan muchos estudiantes a este programa de maestría tiene mucho que ver con la formación que se les da en el pregrado y con la falta de información de los alumnos en cuanto a qué implica cada orientación [...] Con la entrevista, en la selección de aspirantes verificamos sus conocimientos y experiencia en investigación, en ella preguntamos si están trabajando con algún equipo de investigación, les hacemos preguntas como qué es para ellos la investigación, por qué un programa de investigación. El criterio fuerte es la línea de formación y el promedio, no la modalidad de egreso del posgrado [EC1C1].

Cuando llega el aspirante a solicitar el ingreso al programa he notado que por lo menos de diez alumnos nueve nunca han tenido experiencia o acercamiento a la investigación [EC3C3].

Las carreras de diseño se han vuelto muy prácticas, los alumnos no leen nada, y si les das un artículo en inglés mucho menos, hay fallas a nivel de la comprensión lectora en el idioma inglés y eso no cambia mucho en español [EC5C5].

[...] los economistas suelen hacer muchos análisis cuantitativos desde el pregrado y están más familiarizados con la escritura académica, los estudiantes que vienen de otra formación inicial muestran muchas dificultades para la escritura académica [EC2C2].

La gente en diseño y en arquitectura, por ejemplo, saben de métodos de diseño, pero no de métodos de investigación, se les dificulta establecer hipótesis y relaciones entre variables, entre muchas otras cosas $[\ldots]$ dentro de nuestro programa hay muchos diseñadores, pero ellos generalmente no tienen conocimientos sobre cómo abordar una investigación científica, conocen métodos de trabajo en los que se observa más bien un abordaje muy empírico y con poca rigurosidad [...] muchos aspirantes expresan su deseo de conocer, de investigar, pero al mismo tiempo dicen no saber cómo; la carta de exposición de motivos y la entrevista nos dan idea de cómo vienen preparados los alumnos [EC5C5].

En cuanto a los elementos decisivos para el ingreso de los estudiantes a los programas de maestría

Una de las principales causas por las que no aceptamos a los alumnos es por el bajo dominio del idioma inglés y otra carencia es que no saben investigación y aparte no les gusta la investigación por el tipo de carrera de la que vienen [EC3C3]. 
Uno de los requisitos para el ingreso es que el aspirante pertenezca a alguna organización de salud en la que puedas obtener el apoyo institucional, en el caso de los independientes, es decir los que hacen consulta privada, ahí se explora mucho, cómo le vas a hacer, en el momento de la entrevista [...] Uno de los factores de peso para el ingreso es que estén dispuestos a dedicar el tiempo y la estabilidad laboral que tienen [...] se requiere que sean expertos en la parte clínica, si no conocen la enfermedad van a hacer interpretaciones erróneas [EC6C3].

Otro de los tópicos al que se refirieron los coordinadores es el relativo a la normatividad del CONACYT y sus implicaciones en los procesos administrativos y formativos en los programas de maestría, respecto a este tema algunas de las opiniones de los entrevistados se exponen enseguida:

En cuanto a la cantidad de alumnos que podemos aceptar necesariamente tenemos que adaptarnos a las exigencias del CONACYT [...] hay programas que tienen éxito en su interior, pero no cumplen con las exigencias administrativas de CONACYT y eso genera incongruencias que deben resolverse [EC2C2].

El reglamento de la universidad dice que te puedes titular por tesis o proyecto de intervención, pero el PNP solo admite tesis, entonces si le digo a PNP que tengo un programa en el que tengo la opción de titulación por proyecto de intervención me van a decir que no [EC3C3].

CONACYT te pide que busques tu área y no queda más remedio que ubicarte en un área muy general porque las que son más específicas están relacionadas con la tecnología, pareciera que desconocen muchas áreas relacionadas con las ciencias sociales y por eso se cae en generalidades que causan imprecisiones cuando se trata de definir algunos programas de maestría [...] está bien que se establezcan reglas pero no que te dediques a comprobarlas, ellos deberían hacer el seguimiento y yo creo que la formación humana debe ir más allá, sin embargo dejan todo el peso en la coordinación del programa [EC4C4].

Por una parte, las políticas del CONACYT a través de los lineamientos que rigen al PNPC se dirigen principalmente a fortalecer la calidad del posgrado en el país, pero por otra parte parecería que la rigidez de algunos lineamientos y la excesiva flexibilidad de otros, caracterizada por la falta de delimitación en las guías para el funcionamiento de los posgrados, se convierten en un obstáculo para el desarrollo de algunos programas. Al respecto, Arredondo y Navarro (2012) afirman que "el posgrado está regulado en exceso y, a la vez, está insuficientemente regulado” (p. 13).

El último tema que se abordará en esta caracterización contextualizada de los programas de maestría que son muestra de este estudio es el que se refiere a la orientación de las maestrías y su relación con la prosecución de estudios doctorales:

A partir de que se abrió aquí el doctorado, aproximadamente el cincuenta por ciento de los estudiantes continúa sus estudios. Algunos estudiantes desarrollan el gusto por la investigación y otros definitivamente se alejan de este tipo de actividades porque se dan cuenta de que investigar no es lo suyo [EC6C3].

Muchos de los estudiantes que hacen maestrías con énfasis en la investigación continúan su formación con estudios de doctorado a diferencia de los estudiantes que hacen maestrías profesionalizantes [EC2C2]. 
Muchos alumnos al terminar la maestría nos dicen querer continuar en un doctorado, el problema en nuestra disciplina es que los doctorados sólo están en el extranjero [EC5C5].

De acuerdo con las opiniones emitidas por los coordinadores entrevistados en cuanto al interés de los estudiantes por continuar realizando estudios de cuarto nivel, se puede inferir que la maestría tiene una función orientadora en los estudiantes, dado que les permite explorar diversas posibilidades para desarrollarse dentro de sus disciplinas. Por otra parte, las orientaciones de las maestrías parecen configurar el perfil de sus estudiantes y su inclinación hacia la investigación o hacia la aplicación de conocimientos en el campo laboral.

\section{CONSIDERACIONES FINALES}

A manera de síntesis, a continuación se expondrán los elementos que, a partir de los datos emanados de las entrevistas realizadas a coordinadores de distintos programas de maestría, permiten contextualizar y dar significado a las orientaciones de los posgrados más allá de la norma.

En tal sentido, se enuncian de forma comparativa las diferencias y similitudes encontradas en las orientaciones de los posgrados, de acuerdo a los testimonios de los coordinadores como agentes encargados de llevar a cabo los procesos administrativos y en algunos casos formativos que ocurren al interior de los programas de maestría con diferentes orientaciones.

Es necesario reiterar que los lineamientos que definen ambas orientaciones son muy generales, lo que hace que los límites entre los dos tipos de posgrado sean difusos, dando cabida a una interpretación sui géneris del significado de cada orientación. Lo anteriormente expuesto deja en evidencia que la concepción de lo que significa investigar, desde la perspectiva de los coordinadores de los diferentes programas, es variable y guarda relación con la disciplina base a la que pertenecen. Asimismo, aun cuando la orientación de los programas es un referente para diseñar los objetivos de formación que se plantean al interior de los posgrados, no se percibe que tengan debidamente definidos los requisitos para el ingreso y la permanencia de los estudiantes en los diferentes programas, requerimientos que son muy diversos y que carecen de uniformidad entre las orientaciones de los programas de posgrado.

Un problema que quedó en evidencia en este estudio es que la excesiva regulación normativa del PNPC tiende a generar mecanismos de simulación como estrategia para la incorporación y/o permanencia en dicho programa. Se hace necesario entonces profundizar el estudio referente a la normatividad que regula a los posgrados de calidad para crear mecanismos que disminuyan la simulación tanto en los procesos administrativos como de formación. Sin embargo, es importante destacar que la orientación de los programas desde el deber ser (normatividad) es una manera de clarificar los propósitos de los procesos de formación que se dan en su interior. 
La orientación profesionalizante en las maestrías se enfoca en la aplicación de los conocimientos a la práctica en contextos laborales y es flexible en cuanto al tipo de trabajo terminal que debe desarrollar el estudiante para obtener el grado académico de maestro. La actividad formativa más relevante en la orientación profesionalizante es la práctica profesional que se realiza a través de la vinculación con los sectores productivos del país.

Por otra parte, la orientación a la investigación se centra en la formación de investigadores y en la generación de conocimientos, que no necesariamente están en estrecha vinculación con las necesidades de los sectores productivos, puesto que dicha producción es mediada por interrogantes e inquietudes de tipo académico, que suele tener una inclinación más teórica que práctica. Esto, de acuerdo a los testimonios recabados, genera distancia entre lo que se aprende al interior de este tipo de programas y la inserción laboral de sus estudiantes.

Una de las explicaciones de este distanciamiento podría ser que el único tipo de trabajo terminal que se acepta en las maestrías con orientación a la investigación es la tesis, siendo importante señalar que las temáticas abordadas por los estudiantes no están dirigidas necesariamente a resolver problemas prácticos que impacten en la productividad de los sectores que movilizan la economía en el país. Y aunque esto no podría considerarse como una constante, sí es frecuente, de acuerdo a los testimonios de los coordinadores de este tipo de programas, en los que han resaltado que los ejes temáticos de los tesistas deben responder a las necesidades propias de campos académicos más que a la resolución de problemas prácticos propios de ambientes laborales.

$\mathrm{Al}$ vincular las prácticas de formación con las orientaciones de los posgrados, otro factor crucial expuesto por los coordinadores de ambos tipos de maestría es que la forma en la que se enseña a investigar guarda relación directa con la disciplina desde donde se enseña, y esto se evidencia en los procesos de tutoría, que toman diversas formas de acuerdo a la orientación del programa de la maestría y a sus lineamientos internos.

Un ejemplo de ello son los coloquios, que aun cuando son una práctica recurrente para la formación, tanto en la orientación a la investigación como en la profesionalizante, muestran diferencias en cuanto al objetivo para el que son planeados. En los programas con énfasis en la investigación se planea como un espacio en el cual sólo académicos y estudiantes son convocados con la finalidad de disertar sobre los avances de un trabajo de investigación desde una perspectiva teórica y metodológica, mientras que en los programas profesionalizantes son eventos abiertos a organizaciones públicas y privadas en los que se pretende que las investigaciones sean consideradas como aportes para ser implementados en espacios laborales.

Es importante destacar que el objetivo de este artículo fue contextualizar los programas de maestría desde la perspectiva de sus coordinadores como agentes que 
movilizan los procesos administrativos y en muchos casos formativos de los posgrados en cuestión; en tal sentido, es necesario dejar muy claro que la caracterización de los programas que aquí se hace no pretende establecer determinismos ni generalizaciones, ya que los datos empíricos obtenidos a través de las entrevistas hablan de la realidad (que de por sí es dinámica) en un momento determinado.

\section{REFERENCIAS BIBLIOGRÁFICAS}

Arredondo, V. M. y Navarro, D. M. (2012). Un marco normativo y de políticas públicas para el desarrollo del posgrado en México. En M. L. Chavoya Peña y S. Reynaga Obregón (coords). Diversas miradas sobre los posgrados en México. Guadalajara: Universidad de Guadalajara, Centro Universitario de Ciencias Sociales y Humanidades.

Arredondo G., V. M., Pérez R., G. y Morán O., P. (2006). Políticas del posgrado en México. Reencuentro, (45).

Barba, J., González, G. y Barba, R. (2014). Que la fuerza esté contigo: desvelar el lado oscuro de la investigación en educación. Magis. Revista Internacional de Investigación en Educación, 7(14), 125-140.

Cifuentes, R. (2011). Diseño de proyectos de investigación cualitativa. Buenos Aires: Noveduc.

CONACYT-SEP (2015). Marco de Referencia para Evaluación y Seguimiento de Programas de Posgrados Presenciales. Programa Nacional de Posgrados de Calidad. México: Conacyt-Subsecretaría de Educación Superior, versión 6, abril. Recuperado de 2017 http://www.conacyt.mx/index.php/becasy-posgrados/programa-nacional-de-posgrados-decalidad (consulta: 20 feb. 2017).
Del Rincón, D., Arnal, J., Latorre, A. y Sans, A. (1995). Técnicas de investigación en ciencias sociales. Madrid: Dykinson.

García, H. A. P. (2015). El significado multiforme de la orientación profesionalizante en las prácticas de formación de una maestría en educación (tesis doctoral, Universidad de Guadalajara, México).

Hirsch A., A. (2012). Conductas no éticas en el ámbito universitario. Perfiles educativos, 34(SPE), 142-152.

Luchilo, L. (2010). Formación de posgrado en América Latina: políticas de apoyo resultados e impactos. Argentina: Eudeba.

Marsiske, R. (2006). La Universidad de México: historia y desarrollo. Revista Historia de la Educación Latinoamericana, 8, 9-34.

Moreno, B. M. G. (2002). Formación para la investigación centrada en el desarrollo de habilidades. Guadalajara, México: Universidad de Guadalajara.

Orozco, G. y R. González (2011). Una coartada metodológica. Abordajes cualitativos en la investigación en comunicación, medios $y$ audiencias. México: Tintable.

Serna, G. M. y Pérez, M. R. M. (2012). Logros e innovación en el posgrado. Morelia, Michoacán. México: UMSNH, Coordinación General de Estudios de Posgrado.

Universidad de Guadalajara (2004). Reglamento general de posgrado. Guadalajara.

Cómo citar este artículo:

Castro Marcano, S. E. y López Ramírez, E.. (2020). Aproximación a la orientación de las maestrías a través de la voz de sus agentes. IE Revista de Investigación Educativa de la REDIECH, 11, e733. doi: http://dx.doi.org/10.33010/ ie_rie_rediech.v11i0.733. 